\title{
Coupled precipitation and yield strength modelling for non-isothermal treatments of a 6061 aluminium alloy
}

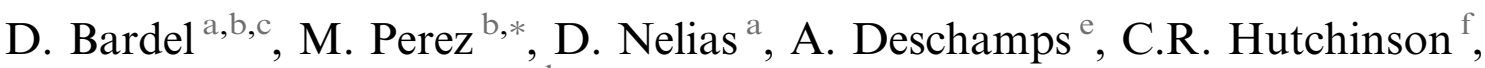 \\ D. Maisonnette ${ }^{\mathrm{a}, \mathrm{d}}$, T. Chaise ${ }^{\mathrm{a}}$, J. Garnier ${ }^{\mathrm{g}}$, F. Bourlier ${ }^{\mathrm{c}}$ \\ ${ }^{\text {a } U n i v e r s i t e ́ ~ d e ~ L y o n, ~ I N S A-L y o n, ~ L a M C o S ~ U M R ~ C N R S ~ 5259, ~} 69621$ Villeurbanne, France \\ ${ }^{\mathrm{b}}$ Université de Lyon, INSA-Lyon, MATEIS UMR CNRS 5510, 69621 Villeurbanne, France \\ ' AREVA NP, 69456 LYON Cedex 06, France \\ ${ }^{\mathrm{d}}$ Mecanium, 69603 Villeurbanne, France \\ e SIMAP, Grenoble INP, CNRS, UJF, BP 75, 38402 St. Martin d'Héres Cedex, France \\ ${ }^{\mathrm{f}}$ Department of Materials Engineering, Monash University, Clayton, Victoria, Australia \\ ${ }^{\mathrm{g}} C E A, D E N, D M N$, SRMA, F-91191 Gif-sur-Yvette, France
}

\begin{abstract}
In age-hardening alloys, high-temperature processes, such as welding, can strongly modify the precipitation state, and thus degrade the associated mechanical properties. The aim of this paper is to present a coupled approach able to describe precipitation and associated yield stresses for non-isothermal treatments of a 6061 aluminium alloy. The precipitation state (in terms of volume fraction and precipitate size distribution) is modelled thanks to a recent implementation of the classical nucleation and growth theories for needle-shaped precipitates. The precipitation model is validated through small-angle neutron scattering and transmission electron microscopy experiments. The precipitation size distribution is then used as an entry parameter of a micromechanical model for the yield strength of the alloy. Predicted yield stresses are compared to tensile tests performed with various heating conditions, representative of the heat-affected zone of a welded joint.
\end{abstract}

Keywords: Yield stress; Precipitation; Age-hardening aluminium alloys; Small-angle neutron scattering; Heat-affected zone

\section{Introduction}

6XXX series aluminium alloys are extensively used for their good combination of specific strength, formability and damage tolerance. These outstanding mechanical properties (compared to pure aluminium) are obtained thanks to a specific heat treatment optimized to obtain the largest density of hardening $\beta^{\prime \prime}$ precipitates (i.e. T6 state) $[1-4]$. However, when complex heat treatments are involved the microstructure can be significantly affected,

\footnotetext{
* Corresponding author.

E-mail addresses: michel.perez@insa-lyon.fr(M. Perez),daniel.nelias@ insa-lyon.fr (D. Nelias).
}

leading to a deterioration of the mechanical properties of the assembly.

As an example, welding of age-hardening alloys leads to drastic change in the precipitation state within the molten zone and the heat-affected zone (HAZ): precipitates may grow, shrink, dissolve and/or coarsen (see, for example, the contribution of Myhr and Grong for 6XXX series $[5,6]$ and Nicolas et al. for 7XXX series [7]). These modifications degrade the mechanical properties (e.g. yield stress) and therefore the operating performance of mechanical parts.

The classical route for understanding the relationship between microstructure and the resulting mechanical properties is based on extensive microstructural and mechanical 


\section{Nomenclature}

\begin{tabular}{|c|c|c|c|}
\hline$a$ & lattice parameter (aluminium) $(\mathrm{m})$ & $r^{*}$ & critical radius of nucleation $(\mathrm{m})$ \\
\hline$b$ & Burgers vector $(\mathrm{m})$ & $r_{i}$ & radius of precipitate in the class $i(\mathrm{~m})$ \\
\hline$D_{\mathrm{i}}{ }^{0}$ & $\begin{array}{l}\text { pre-exponential factor diff. coeff. element } i \\
\left(\mathrm{~m}^{2} \mathrm{~s}^{-1}\right)\end{array}$ & $r^{c}$ & $\begin{array}{l}\text { crit. radius for the shearing/by-passing transi- } \\
\text { tion }(\mathrm{m})\end{array}$ \\
\hline$Q_{\mathrm{i}}^{0}$ & activation energy for diff. coeff. element $i$ & $T$ & temperature $(\mathrm{K})$ or $\left({ }^{\circ} \mathrm{C}\right)$ \\
\hline & $\left(\mathrm{m}^{2} \mathrm{~s}^{-1}\right)$ & $v_{a t}^{p}$ & precipitate mean atomic volume $\left(\mathrm{m}^{3}\right)$ \\
\hline $\bar{F}$ & mean strength of obstacles $(\mathrm{N})$ & $v_{a t}$ & mean atomic volume of the matrix \\
\hline$F(r)$ & strength generated by a precipitate of size $r(\mathrm{~N})$ & $x, y$ & chemistry coefficients for precipitates \\
\hline$F_{b p}$ & obstacle strength for by-passed precipitate $(\mathrm{N})$ & $X_{i}^{0}$ & initial atomic fraction for element $i$ in the matrix \\
\hline$F_{s h}$ & obstacle strength for sheared precipitate $(\mathrm{N})$ & $X_{\mathrm{i}}^{i}$ & at. frac. of $i$ element at the interface ppt/matrix \\
\hline$f_{v}$ & volume fraction of precipitates & $X_{i}^{p}$ & tomic fraction of $i$ element in the precipitate \\
\hline$I$ & scattering intensity & $X_{i}$ & fraction of $i$ element $i$ in the matrix \\
\hline$k$ & strength constant for precipitate shearing calcu- & $Z$ & Zeldovich factor \\
\hline$k$ & lation & $\alpha$ & of atomic volume between matrix and pre- \\
\hline & $i$ & $\beta$ & dislocation line tension \\
\hline$k_{b}$ & Boltzmann constant $(\mathrm{J} / \mathrm{K})$ & $\delta g$ & driving force of nucleation $\left(\mathrm{J} \mathrm{m}^{-3}\right)$ \\
\hline$K_{s}$ & solubility product & $\Delta G^{*}$ & Gibbs energy change for a critical nucleus $(\mathrm{J})$ \\
\hline$L$ & spacing between obstacles in the slip plane (m) & $\Delta p$ & contrast factor $\left(\mathrm{nm}^{-4}\right)$ \\
\hline$l_{i}$ & length of precipitate of class $i(\mathrm{~m})$ & $\Delta \sigma_{p}$ & precipitate contribution to strength $(\mathrm{MPa})$ \\
\hline$M$ & Taylor factor & $\Delta \sigma_{s s}$ & solid solution contribution to strength $(\mathrm{MPa})$ \\
\hline$M_{i}$ & molar mass of element $i(\mathrm{~kg} / \mathrm{mol})$ & $\beta$ & constant related to dislocation line tension $(\mathrm{N})$ \\
\hline$N_{0}$ & number of nucleation sites per unit volume & $\sigma_{y}$ & engineering yield stress (MPa) \\
\hline$N_{i}$ & number density precipitate in class $i\left(\# \mathrm{~m}^{-3}\right)$ & $\sigma_{0}$ & pure aluminium yield stress $(\mathrm{MPa})$ \\
\hline$q$ & scattering vector $\left(\mathrm{m}^{-1}\right)$ & $\Delta \sigma^{b p}$ & by-passed precip. contrib. to yield stress $(\mathrm{MPa})$ \\
\hline$Q_{0}$ & integrated intensity & $\Delta \sigma^{s h}$ & sheared precip. contrib. to yield stress $(\mathrm{MPa})$ \\
\hline$r$ & precipitate radius $(\mathrm{m})$ & $\gamma$ & precipitate/matrix interface energy $\left(\mathrm{J} \mathrm{m}^{-2}\right)$ \\
\hline$r_{p}$ & tip radius of rods $(\mathrm{m})$ & $\mu$ & aluminium shear modulus ( $\mathrm{MPa})$ \\
\hline $\bar{r}$ & precipitate mean radius $(\mathrm{m})$ & $\xi$ & precipitates aspect ratio \\
\hline
\end{tabular}

characterization of samples submitted to non-isothermal treatments, characteristic of the studied process. However, for some processes such as welding, microstructural gradients make the fine microstructural and mechanical characterization very difficult. A coupled approach able to predict both the precipitation evolution and the resulting mechanical properties is an attractive way to overcome this difficulty.

Shercliff and Ashby [8,9] first introduced a process model coupling precipitation and strength in age-hardening alloys. Following their footsteps, many other contributions have been proposed (see the fine review by Simar [10] for the case of friction stir welding). However, the models that have been used are often based on restrictive hypotheses in microstructure and/or micromechanics models and this can limit significantly their generality.

The Kampmann and Wagner numerical model (KWN) [11] was coupled with strain-hardening models to investigate the flow properties in friction stir welded joints $[12,13]$. However, these types of precipitation models were always based on the assumption of spherical precipitates (e.g. [6]), which is often counter to the microstructural observations, especially in the case of Al alloys. Moreover,

the non-spherical shape of precipitates may seriously limit the validity of strength models.

Note that Teixeira et al. [14] used a derivation of the Zener theory [15] to model the growth of plate-shaped precipitates. More costly simulation techniques such as phase field [16] and kinetic Monte Carlo [17] are also particularly suited to model the morphology of non-spherical precipitates (see the review of Hutchinson on precipitation modelling in $\mathrm{Al}$ alloys [18]).

As far as the mechanical properties are concerned, Deschamps and Bréchet [19] showed that a model using only the mean radius and volume fraction of precipitates may be sufficient to estimate the yield stress if a Gaussian distribution is assumed for the precipitate size distribution of spherical precipitates. Later, Myhr et al. [5] proposed to introduce a mean strength to account for a distribution of precipitates. This approach is now widely used $[6,12]$. A recent paper by Bahrami et al. proposed an elegant coupling between a precipitation class model and a strengthening model [20]. However, their precipitation model is based on the integration of the growth equation at constant driving force for precipitation (i.e. parabolic growth: $R \propto t^{1 / 2}$ ), which can give satisfactory results for isothermal treat- 
ments but is obviously unadapted to non-isothermal treatments.

From the experimental point of view, transmission electron microscopy (TEM) has been widely used to validate or calibrate KWN-based models $[5,6,12]$ because it provides information on the chemistry and morphology of precipitates. As far as volume fraction is concerned, small-angle $\mathrm{X}$-ray/neutron scattering (SAXS/SANS) can be used as a complementary bulk analysis technique [19,7,21,22].

In this paper an integrated model is proposed. It aims at describing the yield strength resulting from highly non-isothermal treatments occurring in the HAZ. The modelling approach is based on:

- a robust precipitation model (KWN-type) that accounts for (i) multiclass precipitate size distribution and (ii) non-spherical rod-shaped precipitates (as observed by TEM);

- a yield strength model accounting for (i) the whole precipitate size distribution, (ii) the non-spherical shape of precipitates, (iii) their specific spatial distribution, and (iv) competing shear and bypass strengthening mechanisms.

This approach will be validated on fast heating/cooling experiments performed on a thermomechanical simulator [23]. TEM and SANS will be used to characterize the tensile specimens that have been used at room temperature after several heat treatments to validate the approach.

\section{Materials and treatments}

In this work, 6061 rolled plates (thickness $50 \mathrm{~mm}$ ) are used. Their chemical composition is given in Table 1 .

Samples were first submitted to a solutionizing treatment and water-quenched. The T6 state was obtained via an isothermal treatment performed at $175^{\circ} \mathrm{C}$ for $8 \mathrm{~h}$.

In order to mimic thermal cycles occurring in a HAZ, controlled heating cycles were performed. Each cycle is composed of a heating stage (at constant heating rate) up to a maximum temperature, followed by a cooling stage (natural cooling as in a weld). In real operation, the closer to the weld centre, the higher the heating and the higher is the maximum temperature. In order to study the effects of both heating rate and maximum temperature, two types of cycles were performed:

- at fixed heating rate $\left(15^{\circ} \mathrm{C} \mathrm{s}^{-1}\right)$, achieving maximum temperatures from 200 to $560^{\circ} \mathrm{C}$.

Table 1

Chemical composition of studied 6061 alloy (entire table is used as input for precipitation modelling).

\begin{tabular}{llllllll}
\hline & $\mathrm{Mg}$ & $\mathrm{Si}$ & $\mathrm{Cu}$ & $\mathrm{Fe}$ & $\mathrm{Cr}$ & $\mathrm{Mn}$ & Others \\
\hline wt. $\%$ & 0.93 & 0.61 & 0.28 & 0.26 & 0.2 & 0.12 & 0.123 \\
at. $\%$ & 1.0 & 0.59 & 0.12 & 0.13 & 0.10 & 0.06 & 0.06 \\
\hline
\end{tabular}

- at fixed maximum temperature (close to $400{ }^{\circ} \mathrm{C}$ [23]), with heating rates from 0.5 to $200{ }^{\circ} \mathrm{C} \mathrm{s}^{-1}$.

These thermal cycles, represented in Fig. 1, are typical of thermal cycles occurring in the HAZ.

\section{Characterization techniques}

\subsection{Transmission electron microscopy}

TEM experiments were conducted on a JEOL 2010F microscope operating at $200 \mathrm{kV}$, which belongs to the Centre Lyonnais de Microscopie (CLYM) located at INSA Lyon (France). The samples used for TEM are foils thinned by electropolishing. Energy-dispersive X-ray spectroscopy (EDX) analysis was performed with an Oxford Instruments analyzer, using a nanoprobe (about $3 \mathrm{~nm}$ in diameter) in the microscope to estimate the composition of the precipitates in the T6 state. More details on the TEM characterization can be found in Ref. [23].

\subsection{Tensile tests}

During thermal loadings, displacements of the grips were monitored in order to compensate for thermal expansion of the specimen during heating. Therefore, the experimental setup included a heating device and a MTS mechanical testing machine. Specimens were heated by the Joule effect and the temperature was recorded using a thermocouple spot-welded to the specimen surface. Strains were measured using an extensometer with ceramic tips. The Joule heating device is a power supply, comprising an electrical transformer and a thyristor bridge, providing a continuous current whose intensity is controlled by a thermal controller. Water-cooled cables and clamping systems were used to connect the specimen to the heating device. More details can be found in Ref. [23].

\subsection{Small-angle neutron scattering}

In this material, the $\mathrm{Al}$ matrix and $\mathrm{Mg}$-Si-rich precipitates have very similar atomic numbers. Consequently, the classical and relatively easy-to-use SAXS technique is ineffective. SANS is a related technique that provides information on particle size distribution and volume fraction, but is sensitive to atomic nuclei, making it possible to study $\mathrm{Mg}-\mathrm{Si}$-rich precipitates in $\mathrm{Al}$.

The measurements were performed with the spectrometer D22 at ILL Grenoble on samples extracted from the tensile specimens (see Ref. [23] for more details about tensile tests). The results were treated with the beamline software GRASP, following the same protocol as in Ref. [24].

The detector of the spectrometer D22 is located in a $20 \mathrm{~m}$ discharge tube of, allowing measurements to be made at very small angles. Note that the detector has been placed at two different distances in order to scan a wide range of precipitate radii: a distance of $4 \mathrm{~m}$ (configuration 1) targets 


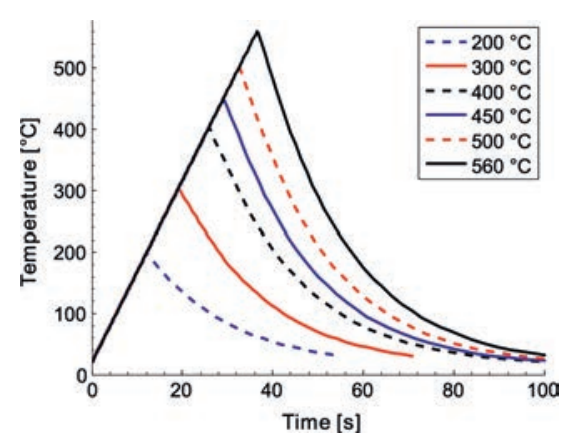

(a)

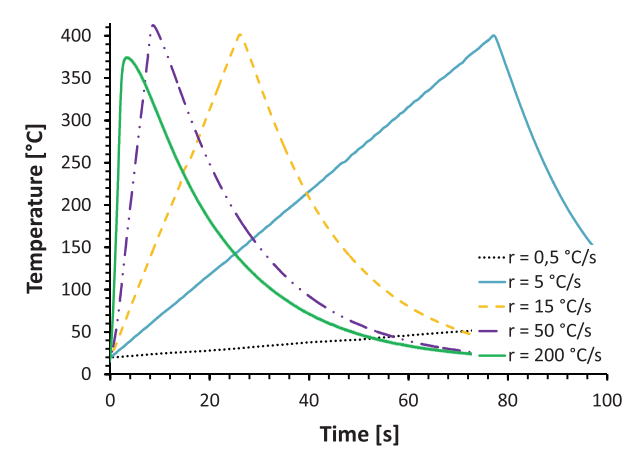

(b)

Fig. 1. Thermal cycles performed on 6061 alloy. The effect of both heating rate (a) and maximum temperature (b) are studied.

precipitates in the range $0.8-12 \mathrm{~nm}$, whereas a larger distance of $17.6 \mathrm{~m}$ (configuration 2 ) has been used to characterize larger precipitates ranging from 3.5 to $45 \mathrm{~nm}$ (see Table 2).

In order to eliminate the scattering caused by the matrix, the scattering spectrum of a sample without precipitates (solution treated, i.e. heated at $560{ }^{\circ} \mathrm{C}$ and quenched - see Ref. [23]) was subtracted from the scattering spectrum of the studied sample. An example of the spectra obtained after this subtraction procedure is shown in Fig. 2 for several microstructural states, along with the curves fitted according to the procedure detailed below.

In this work, according to Ref. [25], a log-normal distribution of ellipsoids containing one axis of revolution is considered. In order to obtain intensity $I$ scattered by a distribution of ellipsoids vs. scattering vector $q$, we make the assumption that orientations are randomly distributed (no texture effect). Thus, one has simply to integrate the scattering contribution of an ellipsoid (major semi-axis $c$, minor semi-axes $a$, angle between major axis and scattering vector $\gamma$ ). Then the precipitate size distribution can be fitted by the following equation [25]:

$$
\left\{\begin{array}{l}
I(q)=\int_{0}^{\infty} I_{\text {ell }}\left(q, r_{\text {ell }}\right) f\left(r_{\text {ell }}\right) d r_{\text {ell }} \\
I_{\text {ell }}\left(q, r_{\text {ell }}\right)=\int_{0}^{\pi / 2} I_{\text {sphere }}\left[q, r_{e q}\left(r_{e l l}, \gamma\right)\right] \sin \gamma d \gamma \\
I_{\text {sphere }}\left(q, r_{e q}\right)=K V^{2}\left[\frac{\sin \left(q r_{e q}\right)-q r_{e q} \cos \left(q r_{e q}\right)}{\left(q r_{e q}\right)^{3}}\right]^{2} \\
r_{e q}\left(r_{e l l}, \gamma\right)=\frac{r_{e l l}}{(0.5 \xi)^{1 / 3}}\left[1+\left(0.25 \xi^{2}-1\right) \cos ^{2} \gamma\right]^{1 / 2}
\end{array}\right.
$$

where $I_{\text {ell }}$ and $I_{\text {sphere }}$ are the ellipsoid and sphere scattered intensities; $r_{\text {ell }}$ is the radius of a sphere of equivalent volume

Table 2

Details of the two configurations used on the D22 line at ILL and the observable radius range.

\begin{tabular}{lll}
\hline Parameters & Configuration 1 & Configuration 2 \\
\hline Wave length $(\mathrm{nm})$ & 0.9 & 0.9 \\
Detector distance $(\mathrm{m})$ & 4 & 17.6 \\
Acquisition time $(\mathrm{min})$ & 17 & 20 \\
Scattering angle $\left({ }^{\circ}\right)$ & $0.68<\theta<9.9$ & $0.18<\theta<2.3$ \\
${\text { Scattering vector }\left(\mathrm{nm}^{-1}\right)}^{\text {Measured radius }(\mathrm{nm})}$ & $0.083<q<1.209$ & $0.022<q<0.276$ \\
\hline
\end{tabular}

$\left(r_{e l l}^{3}=a^{2} c\right) ; r_{e q}$ is the radius of a virtual sphere of equivalent scattering behaviour; $f$ is the size distribution of particles; $K$ is a constant; $V$ is the volume of scattering particle; $\xi$ is the ratio between the large axis $2 c$ and the small semi-axis $a$ $(\xi=2 c / a)$.

These equations are derived by considering that the scattering behaviour of a single ellipsoid obeys the same function as that of a virtual sphere, for which the radius $r_{e q}$ depends on the angle $\gamma$ and the aspect ratio $\xi$. Thus, by introducing $r_{e q}$ into the classical expression for sphere [25] it is possible to identify the precipitate distribution.

Then, the precipitate volume fraction $f_{v}$ was evaluated using the measured integrated intensity $Q_{0}[7]$ :

$Q_{0}=\int_{0}^{\infty} I(q) q^{2} d q=2 \pi \Delta p^{2} f_{v}\left(1-f_{v}\right)$

where $\Delta p$ is the contrast ratio between heterogeneities and the matrix. As noted in Ref. [7], the determination of this parameter is a major challenge because it requires the exact composition of precipitates, which is not straightforward for metastable nanoparticles. According to Andersen et al. [3], the $\beta^{\prime \prime}$ phase has the composition $\mathrm{Mg}_{5} \mathrm{Si}_{6}$, but more complex compositions have been proposed [26].

In this work, the parameter $\Delta p$ was determined from a TEM estimation of $f_{v}$ (see Section 4.1), leading to $\Delta p^{2}=1.16 \times 10^{-8} \mathrm{~nm}^{-4}$.

The combination of TEM and SANS techniques provides, for each investigated state: (i) the mean radius of ellipsoids, (ii) the volume fraction and (iii) the shape factor of $\mathrm{Mg}-\mathrm{Si}$-rich precipitates. The resulting measured mean radius and precipitate volume fraction will be compared to the model in Section 5.

\section{Experimental microstructural results}

\subsection{Initial T6 state}

TEM investigation of the T6 state is given in detail elsewhere [23]. Rods of length $20 \pm 9 \mathrm{~nm}$ and radius $2.0 \pm 0.3 \mathrm{~nm}$ have been observed. Volume fraction has been estimated as $f_{v}=1.6 \pm 0.1 \%$. These data are confirmed by SANS results, provided in Table 3, which shows 


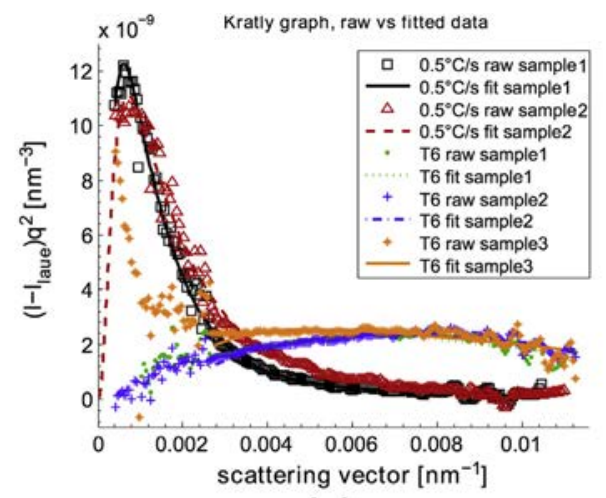

(a)

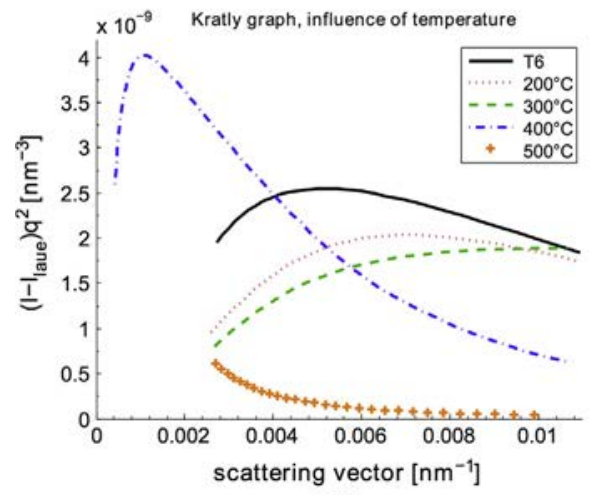

(b)

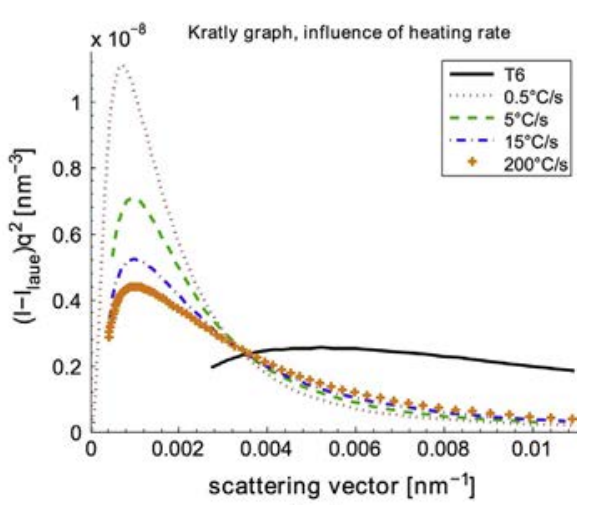

(c)

Fig. 2. (a) Comparison between three $\mathrm{T} 6$ samples and two samples heated at $0.5^{\circ} \mathrm{C} \mathrm{s}^{-1}$. Raw and fitted data are represented. (b) One fitted data for each reached temperature treatment (c) One fitted data for each heating rate treatment.

precipitates of radius $1.7 \pm 0.3 \mathrm{~nm}$ and a ratio $\xi=7 \pm 3$. This state will serve as a reference for the precipitation model.

\subsection{States resulting from non-isothermal treatments}

TEM has been performed on samples submitted to heating rates of $15^{\circ} \mathrm{C} \mathrm{s}^{-1}$ with a maximum temperature of 300 and $400{ }^{\circ} \mathrm{C}$. After heating up to $300^{\circ} \mathrm{C}, \beta^{\prime \prime}$ rods have been observed, with a length of $25 \pm 15 \mathrm{~nm}$ and a radius of $1.5 \pm 0.5 \mathrm{~nm}$. After heating up to $400^{\circ} \mathrm{C}$, fewer and larger precipitates could be observed (assumed to be $\beta^{\prime}$ [23]). Their average length is $120 \pm 50 \mathrm{~nm}$ and their radius is $4 \pm 2 \mathrm{~nm}$.

The SANS results are summarized in Table 3. Maximum heating temperature seems to have an effect on precipitate mean radius from $400{ }^{\circ} \mathrm{C}$, where coarsening may take place. At higher temperature, precipitates eventually dissolve. Note that the measures after heating up to $500{ }^{\circ} \mathrm{C}$ are affected by a very large uncertainty, which is clearly due to the presence of other phases, coarser and more stable than the assumed $\beta^{\prime \prime}-\beta^{\prime}$ phase. Heating rate has moderate effect on precipitate mean radius and volume fraction, except at $0.5^{\circ} \mathrm{C} \mathrm{s}^{-1}$, where coarsening has time to occur during heating, leading to larger precipitates.

As far as aspect ratio is concerned, results obtained by SANS (from Eq. (1)) for different maximum temperatures are summarized in Fig. 3. Considering the uncertainties, it can be reasonably assumed that the precipitate shape factor is constant for all treatments.

\section{Precipitation modelling}

\subsection{KWN model with needle precipitates}

Due to their versatility and simplicity, the classical nucleation and growth theories (CNGTs) have been widely used for modelling the evolution of precipitate size distri-

Table 3

SANS results giving the mean radius, shape factor ration $\xi$ and volume fraction of precipitates. MT and HR stand for "maximum temperature" and "heating rate", respectively. From this data, a constant value of $\xi=11$ has been assumed.

\begin{tabular}{llll}
\hline State & $\begin{array}{l}\text { Mean radius } \\
(\mathrm{nm})\end{array}$ & $\begin{array}{l}\text { Shape factor } \\
\xi=2 c / a\end{array}$ & $\begin{array}{l}\text { Vol. fraction } \\
(\%)\end{array}$ \\
\hline T6 & $1.7 \pm 0.3$ & $7 \pm 3$ & $1.6 \pm 0.1$ \\
MT200 & $1.4 \pm 0.1$ & $12 \pm 6$ & $1.4 \pm 0.1$ \\
MT300 & $1.3 \pm 0.1$ & $20 \pm 10$ & $1.5 \pm 0.1$ \\
MT400 & $8.5 \pm 0.5$ & $15 \pm 4$ & $1.3 \pm 0.2$ \\
MT500 & $3.5 \pm 3.5$ & $5 \pm 5$ & $0.1 \pm 0.1$ \\
HR0.5 & $21 \pm 3$ & $9 \pm 1$ & $1.1 \pm 0.1$ \\
HR5 & $8.1 \pm 0.1$ & $9 \pm 1$ & $1.2 \pm 0.1$ \\
HR15 & $8.5 \pm 0.5$ & $15 \pm 4$ & $1.3 \pm 0.2$ \\
HR200 & $9.4 \pm 1.2$ & $20 \pm 5$ & $1.3 \pm 0.1$ \\
\hline
\end{tabular}




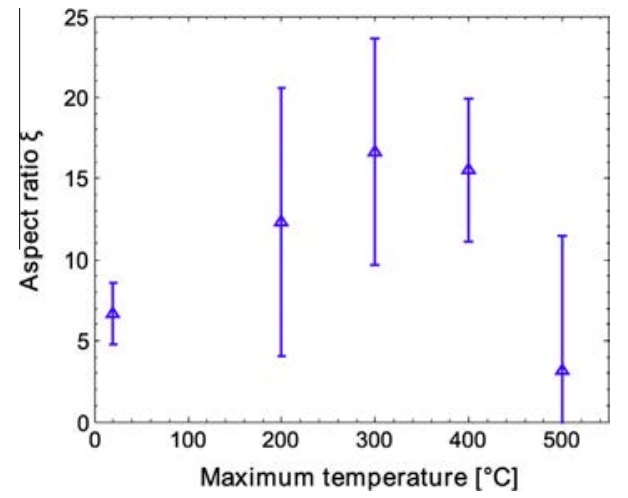

Fig. 3. Aspect ratio $\xi$ from SANS fitting. The error bar is the standard deviation given by the fitting on several samples that have been the same temperature treatment.

butions (see the original contribution of Kampmann and Wagner [11] and examples of implementations [27,28]). In their pioneering contributions, Myhr et al. [5] and Deschamps et al. [19] simplified the complex metastability cascade by accounting for a unique $\beta^{\prime \prime} / \beta^{\prime}$ or $\eta^{\prime}$ spherical precipitate phase. They successfully coupled the precipitation model with a simple strength model. The same approach was also used for non-isothermal heat treatments [6].

The KWN model provides a prediction of the different microstructural phenomena that can occur during various heating kinetics encountered in a welding process: nucleation, growth and coarsening [6]. This model is generally implemented for spherical precipitates [27-29,5-7,12,10]. However, in some cases, including 6XXX series [6], this hypothesis is not acceptable because precipitates have a large aspect ratio (see Fig. 3 and also Ref. [14]).

Several important hypotheses are made here to keep the modelling both tractable and realistic:

- Homogeneous nucleation is considered and elastic energy is assumed to be negligible.

- Only one precipitate kind is considered: an equivalent $\beta^{\prime \prime}-\beta^{\prime}$ precipitate of composition $\mathrm{Mg}_{x} \mathrm{Si}_{y}$. This simplification has been widely used in the literature $[5-7,12]$.

- The needle-shaped precipitates are assumed to comprise a cylinder of length $l-2 r_{p}$ and radius $r_{p}$, ended by two hemispheres of radius $r_{p}$ (see Fig. 4).

- The shape factor of the precipitates $\xi=l / r_{p}$ is considered constant and equal to the one determined in Section 3 (where ellipsoids were considered): $\xi=11$.

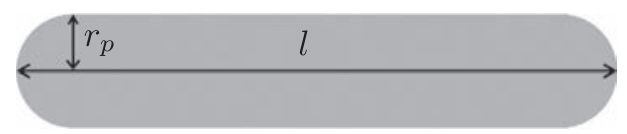

Fig. 4. Needle-shaped precipitates are assumed to comprise a cylinder of length $l-2 r_{p}$ and radius $r_{p}$ ended by two hemispheres of radius $r_{p}$.

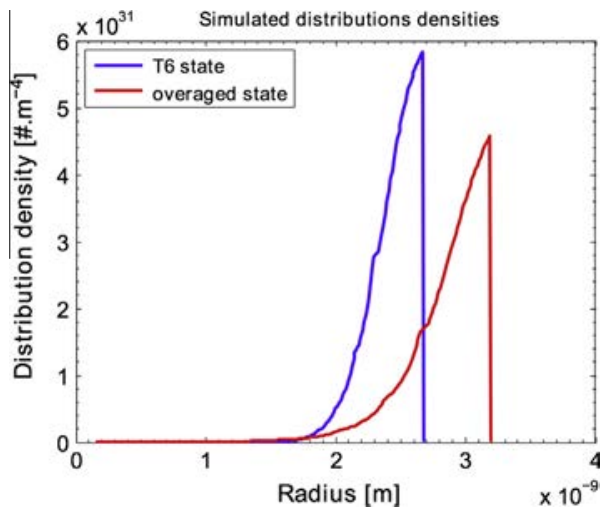

Fig. 5. Precipitate distribution density for the T6 state and an overaged state.

- The model describes precipitate size distribution handling the evolution of $N_{i}$ and $r_{i}$, the number density and radius of class $i$ (see Fig. 5).

Under these assumptions, the classical nucleation theory balances volume and surface forces, expressing the total Gibbs energy change $\Delta G$, due to the presence of a needleshaped precipitate as follows:

$\Delta G=\left(\frac{3 \xi}{4}-\frac{1}{2}\right) \frac{4}{3} \pi r_{p}^{3} \Delta g+\left(\frac{\xi}{2}\right) 4 \pi r_{p}^{2} \gamma$

The critical radius $r^{*}$ is then modified as follows:

$r^{*}=-\frac{2 \gamma}{\Delta g} \frac{2 \xi}{3 \xi-2}$

And so is the energy barrier for nucleation $\Delta G^{*}$ :

$\Delta G^{*}=\frac{16}{3} \pi \frac{\gamma^{3}}{\Delta g^{2}} \frac{2 \xi^{3}}{(3 \xi-2)^{2}}$

This leads to the classical nucleation rate:

$\frac{d N}{d t}=N_{0} Z \beta^{*} \exp \left[-\frac{\Delta G^{*}}{k_{b} T}\right]\left[1-\exp \left(-\frac{t}{\tau}\right)\right]$

where $N_{0}$ is the nucleation site density, $\beta^{*}$ is the condensation rate and $Z$ is the Zeldovich factor, which are given by:

$Z=\frac{v_{a t}^{P}(x+y)}{\pi R^{* 2}(3 \xi-2)} \sqrt{\frac{2 \xi \gamma}{k_{b} T}}$

$\beta^{*}=\frac{4 \pi R^{* 2}}{a^{4}}\left[\frac{X_{\mathrm{Mg}}^{p}}{D_{\mathrm{Mg}} X_{\mathrm{Mg}}}+\frac{X_{\mathrm{Si}}^{p}}{D_{\mathrm{Si}} X_{\mathrm{Si}}}\right]$

Here, $D_{\mathrm{Mg}}$ and $D_{\mathrm{Si}}$ are the $\mathrm{Mg}$ and Si diffusion coefficients, which have the form:

$D_{i}=D_{i}^{0} \exp \left[-\frac{Q_{i}}{R T}\right] \quad i=\mathrm{Mg}, \mathrm{Si}$

The growth equation has been adapted from the needleshaped Zener-Hillert expression [30]:

$\frac{d l}{d t}=1.5 \frac{D_{\mathrm{Mg}}}{2 r_{p}} \frac{X_{\mathrm{Mg}}-X_{\mathrm{Mg}}^{i}}{\alpha X_{\mathrm{Mg}}^{p}-X_{\mathrm{Mg}}^{i}}=1.5 \frac{D_{\mathrm{Si}}}{2 r_{p}} \frac{X_{\mathrm{Si}}-X_{\mathrm{Si}}^{i}}{\alpha X_{\mathrm{Si}}^{p}-X_{\mathrm{Si}}^{i}}$ 
where $X_{\mathrm{Mg}}$ and $X_{\mathrm{Si}}$ are the matrix solute fraction; $X_{\mathrm{Mg}}^{i}$ and $X_{\mathrm{Si}}^{i}$ are the interfacial equilibrium solute fraction; $X_{\mathrm{Mg}}^{p}=x /(x+y)$ and $X_{\mathrm{Si}}^{p}=y /(x+y)$ are the precipitate solute fraction; and $\alpha$ is the ratio between matrix and precipitate mean atomic volume $v_{a t}\left(\alpha=v_{a t}^{M} / v_{a t}^{P}\right)$.

The second term in the original Zener-Hillert expression, namely $1-r^{*} / r_{p}$, was designed to capture the effect of capillarity. It has been removed here because the Gibbs-Thomson effect is more efficiently accounted for by a modification of interfacial concentrations:

$X_{\mathrm{Mg}}^{i}{ }^{x} X_{\mathrm{Si}}^{i}{ }^{y}=K_{s} \cdot \exp \left(\frac{r_{0}}{r}\right)$

where $r_{0}$ is the capillarity length and $K_{S}$ is the solubility. The precipitate shape that has been assumed leads to:

$r_{0}=\frac{4 \xi \gamma v_{a t}^{P}(x+y)}{(3 \xi-2) k_{b} T}$

The solubility product of our precipitates is supposed to have the classical form ( $K_{s}$ is expressed in atomic fraction and $T$ in Kelvin):

$\log _{10} K_{s}=-\frac{A}{T}+B$

where $A$ and $B$ are two constants to evaluate.

Finally, mass balance allows the solute fraction to be updated as precipitation proceeds. We state that the total solute fraction is known and that the solute atom $i$ ( $i$ being either $\mathrm{Mg}$ or $\mathrm{Si}$ ) is distributed either in the matrix or in the precipitate:

$n_{0}^{i}=n_{s s}^{i}+n_{p}^{i}$

The mass balance can be expressed as a function of the precipitate volume fraction $f_{v}$ and the precipitate solute concentration:

$X_{i}=\frac{X_{i}^{0}\left[1+\alpha f_{v}-f_{v}\right]-X_{i}^{p} \alpha f_{v}}{1-f_{v}}$

The precipitate volume fraction is easily calculated knowing the number density $N_{i}$ of all classes $i$ : $f_{v}=\sum_{i} \pi r_{p}^{3}(\xi-2 / 3) N_{i}$

Nucleation and growth equations are implemented in a "Lagrangian-like" model class management software (PreciSo) $[27,28]$ that provides the distribution density of precipitates for a given non-isothermal heat treatment. Details of the implementation are given in Ref. [27,28].

\subsection{Calibration of the precipitation model}

The calibration of the model parameters, namely precipitate solubility ( $A$ and $B$ ) and precipitate interfacial energy $\gamma$, is performed in two steps.

First, the solubility product parameter can be obtained assuming (i) the precipitate volume fraction $f_{v}$ is about $1.6 \%$ for the temperature corresponding to $\mathrm{T} 6$ treatment $(448 \mathrm{~K})$; and (ii) total dissolution of precipitates at $738 \mathrm{~K}$, as shown experimentally by Massardier et al. [31] and confirmed by the first-principles computation of Zhang et al. [32]. These conditions lead to $A=30,750 \mathrm{~K}$ and $B=-16.3$.

Second, precipitate-matrix interfacial energy $\gamma$ can be determined by simulating a T6 treatment from supersaturated solid solution. Indeed, the interfacial energy is directly linked to the nucleation rate, and therefore the number density. We took the target number density of $10^{23} \mathrm{~m}^{-3}$ from the fine characterization of the T6 state of an alloy of similar composition by Donnadieu et al. [33]. It can be seen in Fig. 6 that a value of $\gamma=0.108 \mathrm{~J} \mathrm{~m}^{-} 2$ leads to reasonable values of precipitate number density (Fig. 6b), and of precipitate mean radius (Fig. 6a). Moreover, Fig. $6 \mathrm{c}$ confirms that the chosen interfacial energy leads to an optimal yield strength for the T6 treatment.

The diffusion coefficients for $\mathrm{Mg}$ and $\mathrm{Si}$ have been taken from Ref. [5]. It has been confirmed that these values are in agreement with experimental data [34]. From Vissier et al. [35], the precipitate chemistry has been assumed to be $\mathrm{Mg}_{18} \mathrm{Si}_{10}$ and its mean atomic volume $v_{a t}^{P}=1.92 \times 10^{-29} \mathrm{~m}^{3}$.

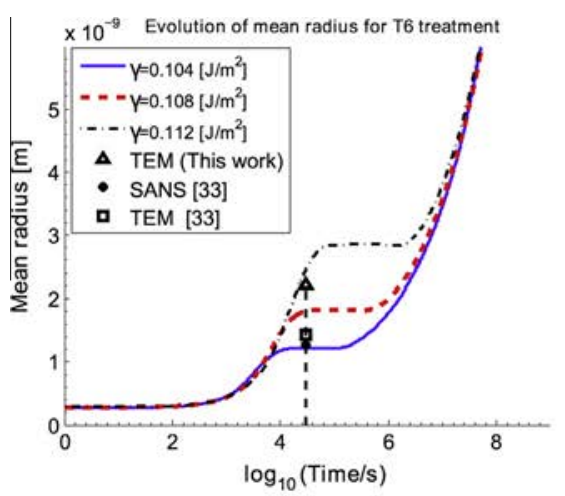

(a)

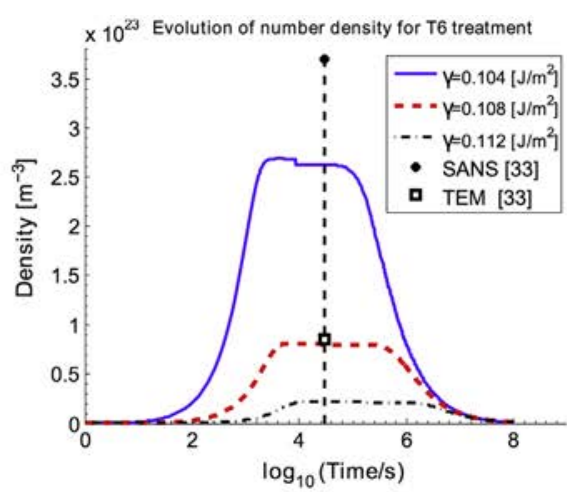

(b)

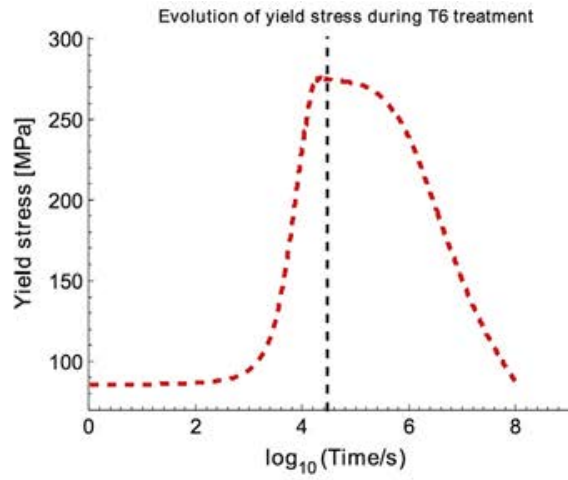

(c)

Fig. 6. Evolution of precipitate mean radius (a), precipitate number density (b) and resulting yield strength (c) during the T6 isothermal treatment from supersaturated solid solution. Yield strength was obtained for $\gamma=0.108 \mathrm{~J} \mathrm{~m}^{-} 2$ using Eq. (17) (see Section 6). 
Table 4

Parameters used for the precipitation model.

\begin{tabular}{lll}
\hline Parameter & Value & Source \\
\hline$x$ & 18 & {$[35]$} \\
$y$ & 10 & \\
$D_{i}^{0}\left(\mathrm{~m}^{2} \mathrm{~s}^{-1}\right)$ & $2.2 \times 10^{-4}$ & {$[5]$} \\
$Q_{i}^{0}\left(\mathrm{~kJ} \mathrm{~mol}^{-1}\right)$ & 130 & {$[5]$} \\
$v_{a t}^{p}\left(\mathrm{~m}^{3}\right)$ & $1.92 \times 10^{-29}$ & {$[35]$} \\
$A(\mathrm{~K})$ & 30750 & This work \\
$B$ & -16.3 & \\
$\gamma\left(\mathrm{J} \mathrm{m}^{-2}\right)$ & 0.108 & Fitted on T6 \\
$a(\mathrm{~m})$ & $4.05 \times 10^{-10}$ & {$[36]$} \\
$v_{a t}^{M}\left(\mathrm{~m}^{3}\right)$ & $1.66 \times 10^{-29}$ & {$[35]$} \\
\hline
\end{tabular}

All parameters of the precipitation model are listed in Table 4.

\subsection{Results}

From the resulting T6 state, all thermal loads representative of welding (Fig. 1) were applied to an initial T6 starting microstructure. Fig. 7 shows the evolution of mean radius and volume fraction as a function of maximum temperature and heating rate.

It can be observed in Fig. 7a and $\mathrm{b}$ that the precipitation state barely evolves for treatments performed below $300{ }^{\circ} \mathrm{C}$. For higher temperatures, coarsening occurs simultaneously with precipitate dissolution, leading to an increase in precipitate mean radius while the volume fraction decreases. Finally, above $520^{\circ} \mathrm{C}$, precipitates are fully dissolved. These predictions are in good agreement with both TEM and SANS experiments.

The model shows a limited effect of heating rate on both precipitate mean radius (Fig. 7) and volume fraction (Fig. 7d), except for the lowest heating rate, for which coarsening occurs during heating, leading to an increase in mean radius. These predictions are, once again, confirmed by experimental TEM and SANS measurements.

Note that the model clearly underestimates the precipitate volume fraction at temperatures ranging from 350 to $500{ }^{\circ} \mathrm{C}$. This is probably due to the fact that the precipitation model only deals with metastable $\beta^{\prime \prime}-\beta^{\prime}$ phase. More stable phases, seen in SANS, are probably appearing in this temperature domain. This is, however, not critical since these coarse phases make a negligible contribution to the yield strength.

In the following section, these microstructural predictions will serve as entry parameters of another model devoted to the estimation of yield stress.

\section{Yield stress estimation}

In this section a model based on the interaction between dislocations and microstructure is proposed in order to cal-

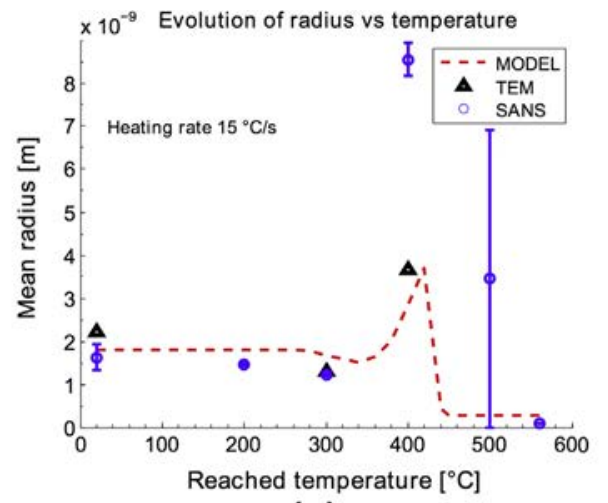

(a)

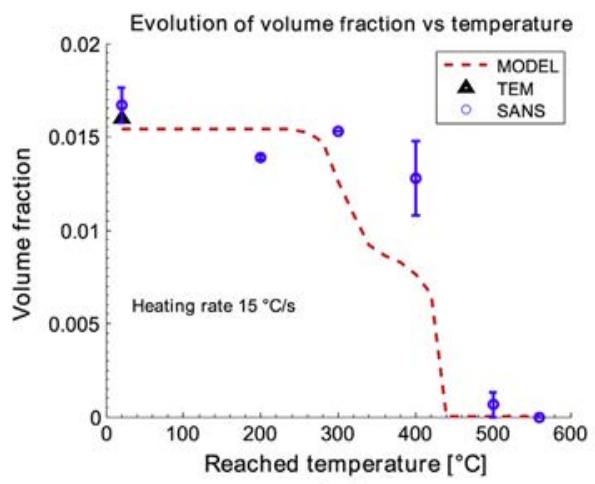

(c)

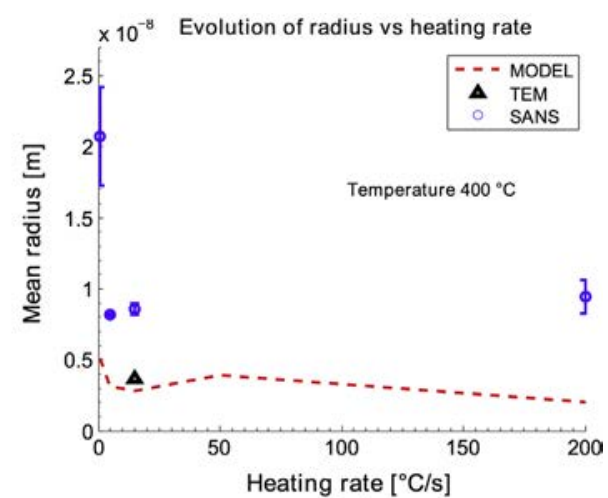

(b)

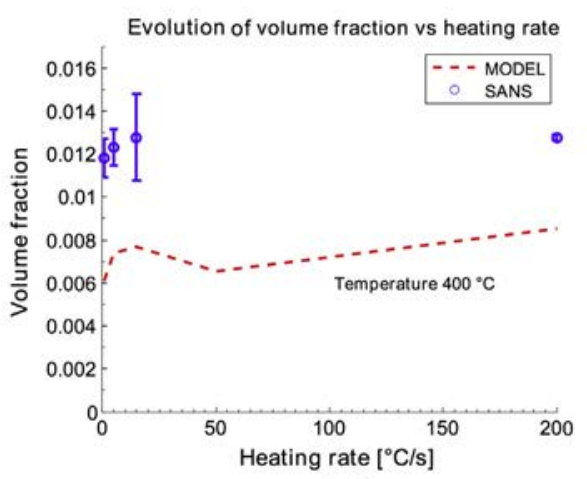

(d)

Fig. 7. Evolution of mean radius (a and b) and precipitate volume fraction (c and d) for various maximum temperatures (heating rate of $15^{\circ} \mathrm{C} \mathrm{s}{ }^{-1}$ : (a) and (c)) and various heating rate (maximum temperature of $400{ }^{\circ} \mathrm{C}$ : (b) and (d)). 
culate the yield stress of AA6061 from the microstructural information presented in Section 3 and 5.

The classical formulation originally proposed by Friedel [37] and improved by Kocks [38] and Deschamps [19] will be reconsidered in order to explicitly take into account the precipitate size distribution provided by the precipitation model and the precipitate morphology (rod-shaped).

The yield strength is supposed to affect (i) the friction stress $\left(\sigma_{0}\right)$, (ii) the solid solution contribution $\left(\Delta \sigma_{s s}\right)$ and (iii) the precipitate contributions $\left(\Delta \sigma_{p}\right)$ that sum up as $[38,19]$ :

$\sigma_{y}=\sigma_{0}+\Delta \sigma_{s s}+\Delta \sigma_{p}$

It is assumed here that grain boundaries and dislocations make a negligible contribution (see Ref. [39]). The friction stress $\sigma_{0}$ of pure aluminium has been set to $\sigma_{0}=10 \mathrm{MPa}$ $[19,10]$.

\subsection{Solid solution}

$\Delta \sigma_{\mathrm{ss}}=\sum_{\mathrm{j}} \mathrm{k}_{\mathrm{j}} . X^{j_{w t}^{2 / 3}}$ is the contribution of solute concentration [37,5]. Here, only $\mathrm{Mg}$ and $\mathrm{Si}$ are taken into account. The $\mathrm{Cu}$ content is very low (see Table 1) and its amount in solid solution is low due to intermetallic and/or several $\mathrm{Q} / \mathrm{QC} / \mathrm{QP}$ phases [40]. Scaling factors $k_{j}$ of $\mathrm{Mg}$ and $\mathrm{Si}$ have been proposed by Myhr et al. [5]: $k_{\mathrm{Si}}=66.3 \mathrm{MPa} / \mathrm{wt} . \% \%^{2 / 3}$ and $k_{\mathrm{Mg}}=29.0 \mathrm{MPa} / \mathrm{wt} . \%^{2 / 3}$.

\subsection{Precipitates}

Precipitates play a major role in the 6061 alloy. In order to quantify their effect on dislocation motion, the balance between Peach-Koehler force, line tension and precipitate-dislocation force $\bar{F}$ needs to be determined. This leads to the expression of the stress at which the precipitates are overcome $[19,38]$ :

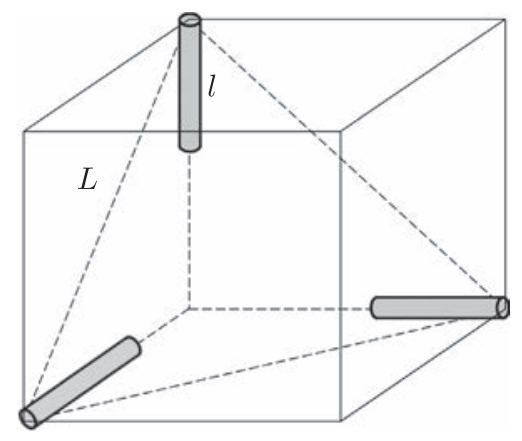

Fig. 8. Disposition of precipitates in the slip plane.

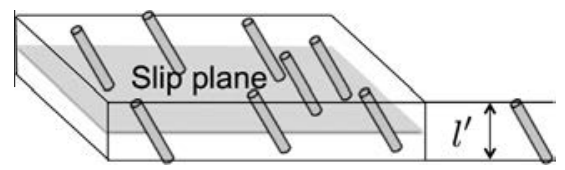

Fig. 9. Intersection of slip plane with rods of length $l$ in the parallelepiped of thickness $l^{\prime}$.
$\Delta \sigma_{p}=\frac{M \bar{F}}{b \bar{L}}=\frac{M}{b \bar{L}} \frac{\sum_{i} N_{i}(r) F(r)}{\sum_{j} N_{j}(r)}$

where $M$ is the Taylor factor $\left(M^{-1}\right.$ is the Schmid factor in a monocrystal), $b$ is the Burgers vector, $F(r)$ is the force acting on precipitate of size $R, N_{i}(r)$ is the precipitate distribution (precipitate number density of class $i$ ) and $\bar{L}$ is the average particle spacing on the dislocation line.

Estimation of $\bar{L}$ In the present case, precipitates are rods of length $l_{i}$ and radius $r_{i}$ aligned in the $\langle 100\rangle$ directions and the shear planes are the $\left\{\begin{array}{lll}1 & 1 & 1\end{array}\right\}$ planes (see Fig. 8). The projection of the rod length on the direction perpendicular to $\left\{\begin{array}{lll}1 & 1 & 1\} \text { planes is: }\end{array}\right.$

$l_{i}=l_{i} \cos ([111],[100])=\frac{l_{i}}{\sqrt{3}}$

On Fig. 8 , it can be seen that the area of the triangle connecting three rods is $A=L^{2} \sqrt{3} / 4$ and the number of precipitates in this plane is $n_{a}=3 \times 1 / 6$. The number of precipitate per unit surface is therefore $N_{a}=n_{a} / A$, which leads to the expression of the average distance between precipitates:

$\bar{L}=\sqrt{\frac{2}{N_{a} \sqrt{3}}}$

Now, we consider a parallelepiped of unit surface parallel to the slip plane and of thickness $l^{\prime}$; the slip plane lies in the middle of the parallelepiped (see Fig. 9). All rods that have their centre within this parallelepiped are intersected by the slip plane, and their number can be expressed as:

$N_{a}=\sum_{i} l_{i}^{\prime} N_{i}=\sum_{i} l_{i} N_{i} / \sqrt{3}$

Leading to:

$\bar{L}=\sqrt{\frac{2}{\sum_{i} l_{i} N_{i}}}$

Sheared vs by-passed precipitates Depending on their size, precipitates can be either sheared or bypassed. For sheared precipitates of size $r$, the force acting on the dislocation is:

$F^{s h}=k \mu b r$

where $k$ is a constant and $\mu$ is the shear modulus of the aluminium. For bypassed precipitates, we have:

$F^{b p}=2 \beta \mu b^{2}$

where $\beta$ is a constant. From these two expressions, we can deduce the critical radius $r^{c}$ from which precipitates are sheared:

$r^{c}=\frac{2 \beta b}{k}$

Bypassed precipitates We assume here that precipitates of radius larger than $r^{c}$ are bypassed. In order to calculate the stress increase due to bypassed precipitates only, we ignore all precipitates smaller than $r^{c}$ (with associated class 
Table 5

Parameters used for yield strength modelling.

\begin{tabular}{lll}
\hline Parameter & Value & Sources \\
\hline$a(\mathrm{~m})$ & $2.86 \times 10^{-10}$ & {$[36]$} \\
$M$ & 2 & {$[12,10,13,19]$} \\
$\beta$ & 0.28 & {$[10,13]$} \\
$\sigma_{0}(\mathrm{MPa})$ & 10 & {$[19]$} \\
$E(\mathrm{GPa})$ & 71.5 & {$[23]$} \\
$v$ & 0.33 & \\
$r^{c}(\mathrm{~nm})$ & 1.8 & From T6 (this work) \\
$k_{M g}\left(\mathrm{MPa} w{ }^{-2 / 3}\right)$ & 29 & {$[5]$} \\
$k_{S i}\left(\mathrm{MPa} w \%^{-2 / 3}\right)$ & 66.3 & {$[5]$} \\
\hline
\end{tabular}

index $i^{c}$ ). The average distance between bypassed precipitates $L^{b p}$ is then defined by:

$L^{b p}=\sqrt{\frac{2}{\sum_{i>i^{c}} l_{i} N_{i}}}$

The yield stress increment $\Delta \sigma^{b p}$ due to bypassed precipitates is then:

$\Delta \sigma^{b p}=\frac{M 2 \beta \mu b^{2}}{b L^{b p}}=\sqrt{2} M \beta \mu b \sqrt{\sum_{i>i^{c}} l_{i} N_{i}}$

Sheared precipitates If rods are sheared, the distance $L^{\text {sh }}$ between obstacles that interact with the dislocation line now depends on the radius of curvature of the dislocation, which itself depends on the applied stress. Under very low stress, the dislocation is almost a straight line and thus does not interact with any precipitates $\left(L^{s h} \rightarrow \infty\right)$. When the stress increases, the dislocation bends until it comes into contact with precipitates.

To estimate the variation of $L^{s h}$, we follow the approach taken by Friedel [37]: if a steady-state flow is reached (at critical shear stress $\tau_{c}$ ), on average the area $\mathscr{A}$ swept by the dislocation after breaking free of an obstacle contains exactly one obstacle. In other words, the product of the area swept by the surface density of precipitates $N_{a}$ is exactly 1: $\mathscr{A} N_{a}=1$.

The area $\mathscr{A}$ can also be estimated from geometrical considerations, leading to effective obstacle spacing (see details in Ref. [41]:

$L^{s h}=\sqrt{\frac{2 \Gamma}{\bar{F} N_{a}}}=\sqrt{\frac{\sqrt{3} \Gamma}{\bar{F}} \bar{L}}$

The average precipitate spacing $\bar{L}$ is estimated using Eq. (22) considering only smaller sheared precipitates. The yield stress increment $\Delta \sigma^{\text {sh }}$ due to precipitate shearing is finally:

$\Delta \sigma^{s h}=M(k \mu)^{3 / 2} \sqrt{\frac{b \sum_{i<i_{c}} l_{i} N_{i}}{2 \sqrt{3} \Gamma}}\left[\frac{\sum_{i<i_{c}} N_{i} R_{i}}{\sum_{i<i_{c}} N_{i}}\right]^{3 / 2}$

The precipitate shearing and bypassing contributions are added according to a quadratic summation law and the result is input into Eq. (17) to obtain the overall yield strength. In the following section, these estimations of yield stress will be compared with experiments.

\subsection{Input data and results}

In Eqs. (29) and (27), several parameters have to be identified (all parameters are given in Table 5).

- The Taylor factor $M$ depends on the texture and the orientation of loading but it has been approximated in several studies. As in Refs. $[19,13,12,10]$, a value of $M=2$ has been chosen.

- The line tension is linked to the propagation mechanisms of dislocations. The parameter $\beta$ depends on the shape and the nature (edge, screw, mixte) of the dislocation. The value of this constant is known to be relatively close to 0.5 [33], nevertheless some authors (e.g. [42]) have reported that 0.25 might be a more realistic estimate. Here, this parameter will be fixed to $\beta=0.28$, as in Refs. $[13,10]$.

- The transition radius $r^{c}$ between shearing and bypassing is chosen to correspond to the mean radius of precipitates for the T6 state, namely $r^{c}=1.8 \mathrm{~nm}$. Note that this value roughly corresponds to the estimated theoretical value of $2.5 \mathrm{~nm}$ suggested by Wang et al. [43].

The yield strength is finally calculated using the full precipitate size distributions obtained from the precipitation model. Comparison with experimental values is shown in Fig. 10 (all parameters are summarized in Table 5).

\section{Discussion}

It should first be noted that the fitting parameters for the precipitation model (namely surface energy and solubility limits) were determined on isothermal $\mathrm{T} 6$ treatments and were kept unchanged. Moreover, for the calculation of yield stress, all parameters have been estimated from the literature, except the critical radius, which has been set to the value observed (and modelled) for the T6 state. The T6 treatment indeed corresponds to the maximum yield stress for this alloy as can be observed in Fig. 6c.

Concerning the effect of maximum temperature, as expected from the microstructural characterization, thermal treatments performed below $250{ }^{\circ} \mathrm{C}$ do not lead to any change in yield strength. From $300^{\circ} \mathrm{C}$, the yield stress decreases until it reaches the value corresponding to the complete dissolution of precipitates. This effect is clearly associated with the coarsening of the precipitates at low heating rates, leading to a fall in strength. Once again, this is well captured by the model (see Fig. 7a).

The mechanical properties also depend on the heating rate: the slower the heating rate, the lower the yield stress. This effect is clearly associated with the coarsening of the precipitates occurring at low heating rates, leading to a drop in strengthening. Once again, this is due to coarsening of precipitates, predicted by the model (see Fig. 7b).

The results presented in Fig. 10 show a very good correlation with experimental tensile tests. It can be observed 


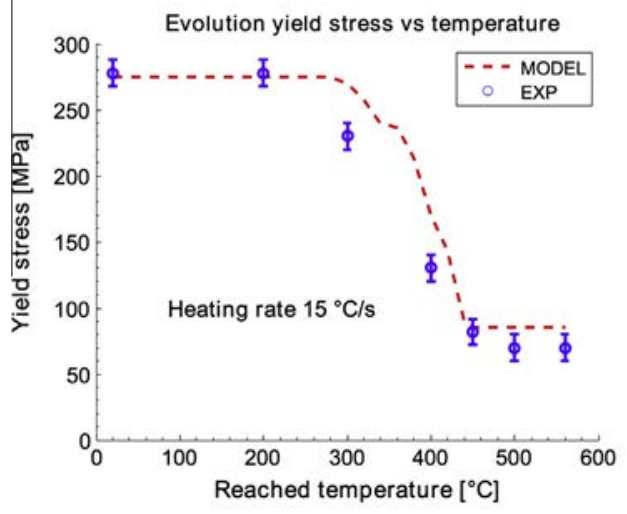

(a)

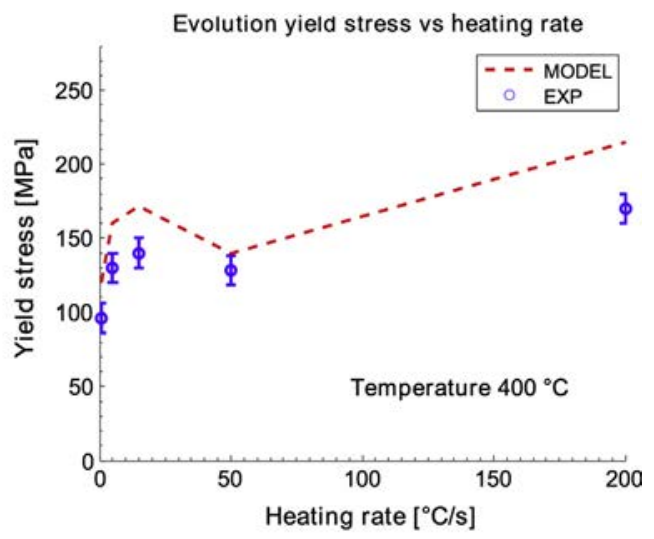

(b)

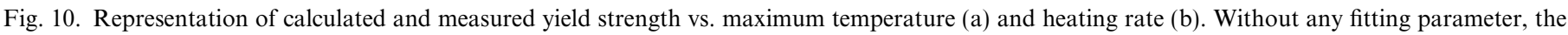
model reproduces all trends observed experimentally.

that this model provides a remarkably good agreement for all experimental conditions.

This agreement validates the coupled approach. Knowledge of the complete precipitate size distribution is indeed required (i) to account for non-isothermal heat treatments (as shown in Refs. $[27,28,44]$ ); and (ii) to predict the yield stress accurately. Moreover, the mean distance between precipitates that is necessary to predict the yield stress also requires knowledge of the complete distribution of obstacles (which is not described only by the mean radius and volume fraction).

Note that, even if the precipitation model underestimates the precipitate volume fraction for temperatures ranging from 350 to $500{ }^{\circ} \mathrm{C}$, this has little consequence for the mechanical properties. This tends to confirm the former assumption that precipitates observed in this range may contain coarser and more stable phases, which make a negligible contribution to strengthening.

The agreement between experiments and models for both precipitation and yield strength models proves that accounting for the non-spherical shape of the precipitates was necessary, especially for such shape factors $(\approx 10)$.

This provides new perspectives for the modelling of complex thermal treatments (e.g. welding). Note that these treatments could also involve nucleation.

\section{Conclusions}

A coupled precipitation/strengthening model was developed to describe the microstructural and strength evolutions that occur during non-isothermal treatments of a 6061 alloy with a starting T6 state.

A recently developed precipitation model has been improved to account for non-spherical precipitates. It provides particle size distribution for any kind of thermal treatment and the mechanical model uses this information as input parameters to calculate the yield strength.
A combination of global (SANS) and local (TEM) characterization techniques was used to characterize the microstructure of the Al alloy.

The precipitation model was calibrated on an isothermal treatment (T6 treatment) state. It reproduces microstructural evolutions occurring during non-isothermal treatments.

A classical model for the prediction of yield stress has been developed to account for non-spherical distribution of obstacles. This model contains fitting parameters (interfacial energy and solubility limit) and other parameters chosen from the literature.

The resulting mechanical properties are successfully compared with tensile tests and show that this model can provide good results for a wide range of non-isothermal heat treatments.

This coupled approach can now be straightforwardly used to predict the microstructural heterogeneities in the HAZ of a weld, and their mechanical behaviour that these cause.

\section{Acknowledgements}

The authors gratefully acknowledge Areva and the French Atomic Energy Commission (CEA) for industrial and financial support. The staff of the D22 beam line of Laue Langevin Institute is thanked for technical help. C.R.H. gratefully acknowledges the support of INSA Lyon in the form of a visiting professorship during 2012. T.C. would like to acknowledge the AREVA-SAFRAN chair for partial funding.

\section{References}

[1] Guinier A, Lambot H. Comput Rend Acad Sci 1948;227:74.

[2] Lutts A. Acta Metall 1961;9(6):577-86.

[3] Andersen J, Zandbergen HW, Jancen J, Traeholt C, Tundal U, Reiso O. Acta Mater 1998;46:3283-98. 
[4] Lorimer GW. Precipitation in aluminium alloys. In: Precipitation processes in solids. Warrendale (PA): Met. Soc. of AIME; 1978. p. 87-119.

[5] Myhr OR, Grong Ø, Andersen SJ. Acta Mater 2001;49:65-75.

[6] Myhr OR, Grong ØG, Fjaer HG, Marioara CD. Acta Mater 2004;52:4997-5008.

[7] Nicolas M, Deschamps A. Acta Mater 2003;51:6077-94.

[8] Shercliff H, Ashby M. Acta Metall Mater 1990;38(10):1789-802.

[9] Shercliff H, Ashby M. Acta Metall Mater 1990;38(10):1803-12.

[10] Simar A, Bréchet Y, de Meester B, Denquin A, Gallais C, Pardoen T. Prog Mater Sci 2012;57:95-183.

[11] Kampmann R, Wagner R. Kinetics of precipitation in metastable binary alloys - theory and application to Cu-1.9 at\% Ti and Ni-14 at $\%$ Al. In: Decomposition of alloys: the early stages. Oxford: Pergamon Press; 1984. p. 91-103.

[12] Simar A, Bréchet Y, de Meester B, Denquin A, Pardoen T. Acta Mater 2007;55:6133-43.

[13] Gallais C, Denquina A, Bréchet Y, Lapasset G. Mater Sci Eng A 2008;496:77-89.

[14] da Costa Teixeira J, Cram DG, Bourgeois L, Bastow TJ, Hill AJ, Hutchinson CR. Acta Mater 2008;56:6109-22.

[15] Hillert M, Hoglund L, Agren J. Acta Mater 2003;51:2089-95.

[16] Vaithyanathan V, Wolverton C, Chen LQ. Acta Mater 2004;52:2973-87.

[17] Clouet E, Nastar M, Sigli C. Phys Rev B 2004;69:064109.

[18] Hutchinson CR. Modeling the kinetics of precipitation in aluminium alloys. In: Fundamentals of aluminium metallurgy: production, processing and applications. Cambridge: Woodhead Publishing; 2010. p. 422-67.

[19] Deschamps A, Bréchet Y. Acta Mater 1999;47:293-305.

[20] Bahrami A, Miroux A, Sietsma J. Met Mater Trans A 2012;43:4445-53.

[21] Dumont M, Lefebvre W, Doisneau-Cottignies B, Deschamps A. Acta Mater 2005;53:2881-92.
[22] Dumont M, Steuwer A, Deschamps A, Peel M, Withers PJ. Acta Mater 2006;54:4793-801.

[23] Maisonnette D, Suery M, Nelias D, Chaudet P, Epicier T. Mater Sci Eng A 2011;528:2718-24.

[24] Deschamps A, Danoix F, Geuser FD, Epicier T, Leitner H, Perez M. Mater Lett 2011;65:2265-8.

[25] Deschamps A, Geuser FD. J Appl Crystallogr 2011;44:343-52.

[26] Marioara CD, Andersen SJ, Jansen J, Zandbergen HW. Acta Mater 2001;49:321-8.

[27] Perez M, Dumont M, Acevedo-Reyes D. Acta Mater 2008;56:2119-32.

[28] Perez M, Dumont M, Acevedo-Reyes D. Acta Mater 2008;57:1318.

[29] Perez M. Scripta Mater 2005;52:709-12.

[30] Hillert M. Jernkont Ann 1957;141(11):757-89.

[31] Massardier V, Epicier T, Merle P. Acta Mater 2000;48:2911-24.

[32] Zhang H, Wang Y, Shang SL, Ravi C, Wolverton C, Chen LQ, et al. Comput Coupl Phase Diagr Thermochem 2010;34:20-5.

[33] Donnadieu P, Roux-Michollet M, Chastagnier V. Philos Mag A 1999;79:1347-66.

[34] Du Y, Chang Y, Huang B, Gong W, Jin Z, Xu H, et al. Mater Sci Eng A 2003;363:140-51.

[35] Vissers R, van Huis MA, Jansen J, Zandbergen HW, Marioara CD, Andersen SJ. Acta Mater 2007;55:3815-23.

[36] Ravi C, Wolverton C. Acta Mater 2004;52:4213-27.

[37] Friedel J. Dislocations. Oxford: Pergamon Press; 1964.

[38] Kocks U, Argon A, Ashby M. Prog Mater Sci 1975;19:1.

[39] Starink MJ, Deschamps A, Wang SC. Scripta Mater 2008;58:377-82.

[40] Cayron C, Buffat P. Acta Mater 2000;48:2639-53.

[41] Esmaeili S, Lloyd D, Poole WJ. Acta Mater 2003;51:2243-57.

[42] Brown L, Stobbs W. Philos Mag 1971;23:1185-99.

[43] Wang X, Poole WJ, Esmaeili S, Lloyd DJ, Embury JD. Metall Mater Trans A 2003;34A:2913-24.

[44] Deschamps A, Perez M. Comput Rend Acad Sci 2010;11:236-44. 